\title{
Statistical properties of a modified standard map in quantum and classical regimes
}

\author{
Piotr Pepłowski · Piotr Weber
}

Received: 12 February 2018 / Accepted: 10 December 2018 / Published online: 19 December 2018

(C) The Author(s) 2018

\begin{abstract}
We present a model - a modified standard map. This model has interesting properties that allow quantum-classical correspondences to be studied. For some range of parameters in the classical phase space of this model, there exist large accelerator modes. We can create a family of maps that have large accelerator modes.
\end{abstract}

Keywords Low-dimensional chaos · Accelerator modes · Quantum chaos · Husimi function

\section{Introduction}

We consider a nonlinear, chaotic, Hamiltonian system that mathematically describes a periodically perturbed two-dimensional rotator. Depending on the form of the perturbation, we can get a variety of dynamical properties of the system. If the perturbation is periodic and realized by short kicks, modeled as a periodic Dirac

P. Pepłowski

Department of Quantum Physics, Faculty of Physics, Astronomy and Informatics, Nicolaus Copernicus University, ul. Grudziądzka 5, 87-100 Toruń, Poland e-mail: peplow@fizyka.umk.pl

\section{P. Weber $(\varangle)$}

Atomic and Optical Physics Division, Department of Atomic, Molecular and Optical Physics, Faculty of Applied Physics and Mathematics, Gdańsk University of Technology, ul. G. Narutowicza 11/12, 80-233 Gdańsk, Poland

e-mail: piotr.weber@pg.edu.pl $\delta$-function: $\sum_{n} \delta(t-n T)$, then it is possible to integrate it over one period in time and, in the end, obtain a discrete map [1-3]. One of the most famous models of this type was introduced by Chirikov [4], and it is known as a standard or Chirikov map (SM). In SM, perturbation is realized by periodic kicks in time and by a sine function of the position. This model is crucial from a theoretical point of view, as a quite simple model that exhibits Hamiltonian chaos. It has found many applications in accelerator physics, plasma physics, and solid-state physics. In this article, we investigate properties of the modified standard map (MSM) introduced in [5] and its quantum counterpart. In this model, a two-dimensional rotator is also perturbed in time by force modeled by a periodic Dirac $\delta$-function, but we changed the functional dependency of this perturbation on position. The Hamiltonian function of our model has the following form:

$H(P, Q, t)=\frac{P^{2}}{2 I}+K V(Q) \sum_{n=-\infty}^{+\infty} \delta(t-n T)$,

where $P$ is the momentum, $I$ is moment of inertia, $t$ is time, $T$ is the period of the force, and $V(Q)$ is a periodic function-external potential. We can write the Hamilton equations for the dynamics of our model as

$$
\begin{aligned}
\frac{\mathrm{d} P}{\mathrm{~d} t} & =K f(Q) \sum_{n=-\infty}^{\infty} \delta(t-n T) \\
\frac{\mathrm{d} Q}{\mathrm{~d} t} & =\frac{P}{I},
\end{aligned}
$$


where $f(Q)$ and $V(Q)$ are related by the standard formula:

$V(Q)=-\int_{0}^{Q} f(\tilde{Q}) \mathrm{d} \tilde{Q}$.

In the case of SM, perturbation depends on position $Q$ according to the formula $V(Q)=\cos Q$.

For the models considered in this article, the socalled accelerating modes (AMs) emerge $[4,6,7]$ in phase space for a wide range of parameters. There are areas of the phase space where momentum increases (or decreases) approximately linearly in time. In the case of SM, there are two kinds of AM. However, areas of $\mathrm{AM}$ in $\mathrm{SM}$ are quite small. Using our modifications, we can create AMs that are several times larger and parametrically control their areas. We can also propose modification when only one kind of AMs exist with quite large areas. Simultaneously, trajectories of the area outside the accelerator modes can "stick" to their boundaries and follow a linear evolution for a very long time. This phenomenon leads to statistically interesting results in the form of power-like tails in the momentum distribution function [8].

In this paper, we study the classical and quantum dynamics of a system, for a particular range of parameters for which AMs exist. Because AMs have an important practical interpretation [9-12], we hope that our results may be relevant in this area.

\section{Modified standard map}

Equations (2) can be integrated over time, and this leads to classical discrete dynamical system, which is a modification of SM:

$$
\begin{aligned}
P_{n+1} & =P_{n}+f\left(Q_{n}\right) \\
Q_{n+1} & =Q_{n}+P_{n+1} \bmod 2 \pi,
\end{aligned}
$$

where $Q_{n}$ is a position at the $n$th step, $P_{n}$ represents momentum in the $n$th step, and $f(Q)$ is a piecewise linear continuous periodic function:

$$
f(Q)=\left\{\begin{array}{ll}
a_{1} Q+b_{1} & 0 \leq Q \leq \Theta_{1} \\
a_{2} Q+b_{2} & \Theta_{1}<Q<\Theta_{2} \\
a_{3} Q+b_{3} & \Theta_{2} \leq Q<2 \pi
\end{array} .\right.
$$

Let us recall that the iteration steps of the map correspond to equal time increments. It is also assumed that the mean value of the momentum increment $\left\langle P_{n+1}-\right.$ $\left.P_{n}\right\rangle$ is equal to zero. We additionally require that there exists a stable AM with a center at $Q=\Theta_{1} / 2$, and this implies the following equation:

$a_{1} \frac{\Theta_{1}}{2}+b_{1}=2 \pi$.

These assumptions allow one to express parameters $a_{i}, b_{i}, i=1,2,3$ as a linear function of one parameter, say $a_{1}$. Moreover, one can easily check that the solution $\left(Q_{n}, P_{n}\right)=\left(\Theta_{1} / 2,2 n \pi\right)$ is an elliptic point for $a_{1} \in(-4,0)$, and therefore, only for this range of values, there exists a stable AM.

With this modification, it is much easier to investigate the quantum counterpart of the classical map, especially effects like tunneling from the AM or the stickiness of its boundary (see Fig. 1). Using a proper choice of parameters, the area of AM in the phase space is relatively large (see Fig. 2). It should be noted that MSM as well as SMs is obtained from Hamilton equations in accordance with the assumption that the time dependence of the acting force is a periodic Dirac $\delta$ function; thus, we are considering the so-called kicked model. This also allows the exact form of the quantum map to be obtained. However, in the case of continuous and smooth time dependence in the quantum description, one can apply the well-known Floquet Hamiltonian.

\section{Quantum map}

In this section, we construct a quantum counterpart of Equations (4). With this aim, we start from Schrödinger's equation

$i \hbar \frac{\partial}{\partial t} \psi(Q, t)=\hat{H}(Q, t) \psi(Q, t)$,

with the Hamilton operator in the following form:

$$
\hat{H}(Q, t)=-\frac{\hbar^{2}}{2 I} \frac{\partial^{2}}{\partial Q^{2}}+K V(Q) \sum_{n=-\infty}^{+\infty} \delta(t-n T),
$$

where the second term represents periodic kicks with period $T$. Due to the form of the Hamiltonian, the evolution operator from time $t=0$ to $t=T$ may be written as a product of two simple operators:

$$
\begin{aligned}
\hat{U}(T, 0)= & \exp \left(-\frac{i}{\hbar} K V(Q)\right) \\
& \times \exp \left(-\frac{i}{\hbar} T \hat{H}_{0}\right)=\hat{U}_{Q} \hat{U}_{P}
\end{aligned}
$$



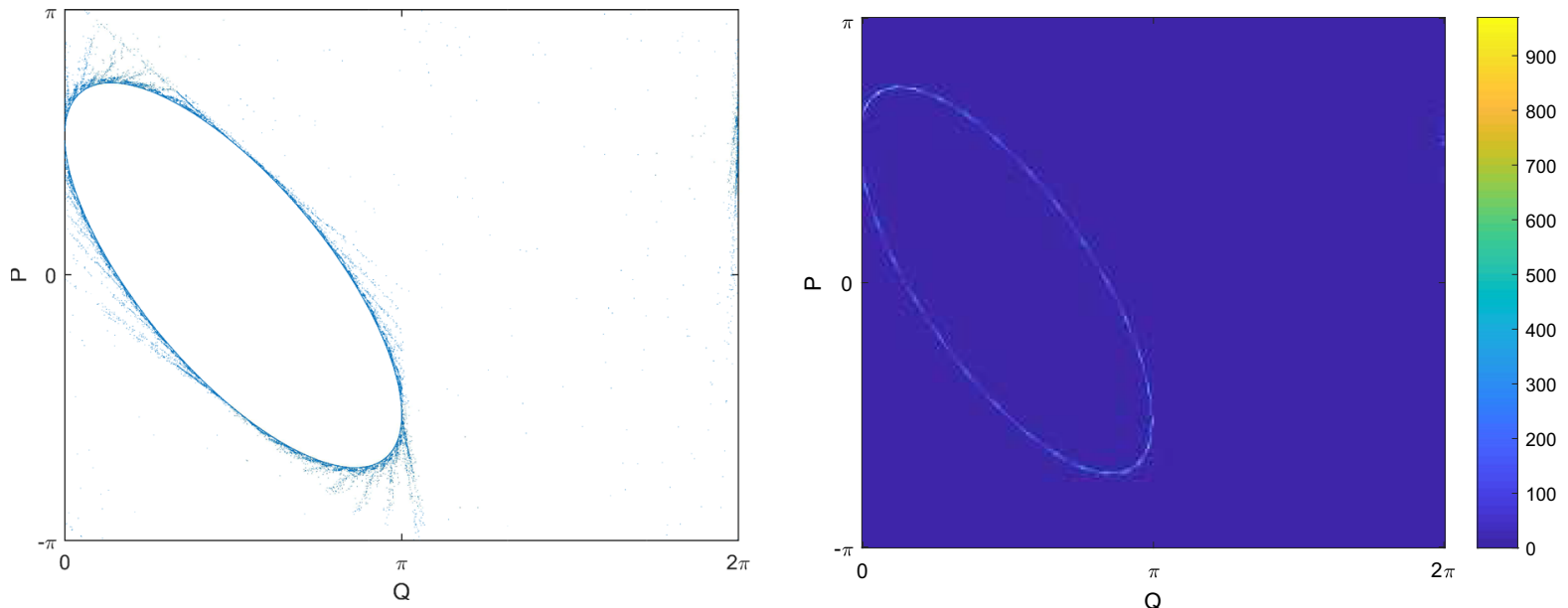

Fig. 1 Stickiness of AM. 105449 points of trajectories starting in chaotic part of phase space whose momentum increased approximately $5000 \times 2 \pi$ after 5000 iterations. In the figure, momentum is plotted modulo $2 \pi$

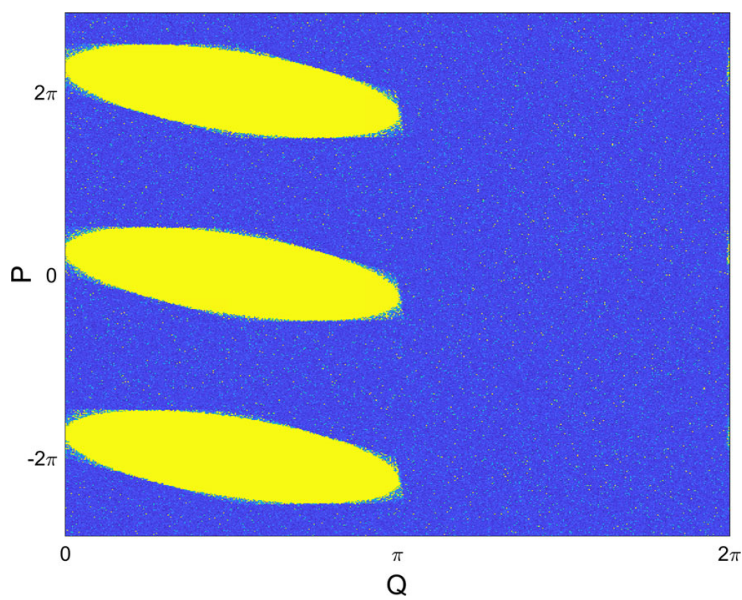

Fig. 2 Points of trajectories in the phase space of MSM for parameters when AMs exist after a large number of iterations. Points belonging to AM escape to the large value of momentum

where $\hat{H}_{0}$ is the operator of free rotation and we assume that we start from free rotation. It should be noted that each operator in the above equation is a simple multiplication in the corresponding representation: $\hat{U}_{Q}$ is a multiplication in position representation, and $\hat{U}_{P}$ is a multiplication in momentum representation. In our case, the phase space is a cylinder with periodicity in variable $Q$; therefore, we have discrete momentum representation with $P_{m}=m \hbar, m=$ $\ldots,-2,-1,0,1,2, \ldots$. In numerical computations, we take into account only a finite number of $P$ values say $P_{m}=-N \hbar, \ldots,(N-1) \hbar$ and conse- quently a finite number of discrete $Q$-values: $Q_{j}=$ $2 \pi j /(2 N), j=0,1,2, \ldots, 2 N-1$. The transitions between both representations are given by the discrete Fourier transform and its inversion:

$$
\begin{aligned}
& \tilde{\psi}\left(P_{m}, t\right)=\sum_{n=0}^{2 N-1} \psi\left(Q_{n}, t\right) \mathrm{e}^{-i 2 \pi n m /(2 N)} \\
& \psi\left(Q_{n}, t\right)=\frac{1}{2 N} \sum_{m=-N}^{N-1} \tilde{\psi}\left(P_{m}, t\right) \mathrm{e}^{i 2 \pi n m /(2 N)}
\end{aligned}
$$

In the classical map (4), the area $C_{n}=[0,2 \pi) \times$ $[(2 n-1) \pi,(2 n+1) \pi)$ is an elementary cell, that is, if the point $\left(Q_{0}, P_{0}\right)$ is transformed into $\left(Q_{1}, P_{1}\right)$ then $\left(Q_{0}, P_{0}+2 k \pi\right)$ is transformed into $\left(Q_{1}, P_{1}+2 k \pi\right)-$ i.e., the classical mapping is invariant with respect to the translation of $2 k \pi$ in momentum. The number of quantum states that belong to such an elementary cell is strictly determined by the value of constant $\hbar$. However, we can treat $\hbar$ as a parameter that may take any positive value. Then, the number of quantum states belonging to the elementary cell can be calculated as $2[\pi / \hbar]+1$, where symbol [.] means an integer part of the fraction $\pi / \hbar$ or as $2 \pi / \hbar$, when multiples of $\hbar$ are equal to $\pi$.

\section{Classical and quantum correspondence}

In the studies of relationships between classical and quantum dynamics, it is useful to represent a quantum state in phase space. Such possibility gives us a Wigner function and Husimi-Kano function. In our case, the 
phase space has a cylindrical geometry and position is a cyclic variable. For that geometry of phase space, the Wigner function can be written in the following way [13]:

$$
\begin{aligned}
W_{\psi}\left(Q, P_{n}\right)= & \frac{1}{\pi \hbar} \int_{-\frac{\pi}{2}}^{+\frac{\pi}{2}} \exp \left(-2 \frac{i}{\hbar} P_{n} Q^{\prime}\right) \\
& \times \psi^{*}\left(Q+Q^{\prime}\right) \psi\left(Q-Q^{\prime}\right) \mathrm{d} Q^{\prime},
\end{aligned}
$$

and the Husimi function on cylinder is defined by the following formula $[14,15]$ :

$$
\begin{aligned}
& H_{\psi}\left(P_{n}, Q\right)=\mid \hbar \sum_{m=-\infty}^{+\infty}\left(\frac{s}{\pi \hbar^{2}}\right)^{\frac{1}{4}} \\
& \quad \times\left.\exp \left(-\frac{s\left(P_{m}-P_{n}\right)^{2}}{2 \hbar^{2}}+\frac{i Q\left(P_{m}-P_{n}\right)}{\hbar}\right) \psi\left(P_{m}\right)\right|^{2},
\end{aligned}
$$

We analyze the quantum evolution of a state given by:

$$
\begin{aligned}
\psi(Q, 0)= & \frac{1}{(2 \pi D)^{\frac{1}{4}}} \exp \left(-\frac{\left(Q-Q_{0}\right)^{2}}{4 D}\right) \\
& \times \exp \left(i \frac{P_{0}}{\hbar} Q\right),
\end{aligned}
$$

where $D \in \Re$ (real number), $Q_{0}$ and $P_{0}$ denote the mean value of position and momentum, respectively. When we choose the numbers $Q_{0}$ and $P_{0}$, we can localize the maximum of the Husimi function in various regions of the classical phase space both in accelerator modes and in the chaotic part of phase space. We can also transform initial Gaussian states to obtain a rotated Husimi or Wigner function. Such a situation is presented in Fig. 3 where both functions are drawn as contours inside the AM which has the shape of a rotated ellipse.

This initial Gaussian state can be obtained by rotating the Wigner function (13) by the angle $\varphi$ and finding its wave function $\psi_{\varphi}(Q)$. In the case of the plane as a phase space, one obtains the following form of this function:

$$
\begin{aligned}
\psi_{\varphi}(Q)= & \left(\frac{\alpha}{\pi \hbar\left(\alpha^{2} \cos ^{2} \varphi+\sin ^{2} \varphi\right)}\right)^{1 / 4} \\
& \times \exp \left\{-\frac{Q^{2}}{4 \Sigma^{2}}\right\}
\end{aligned}
$$

where

$\alpha=\frac{2 D}{\hbar}$,
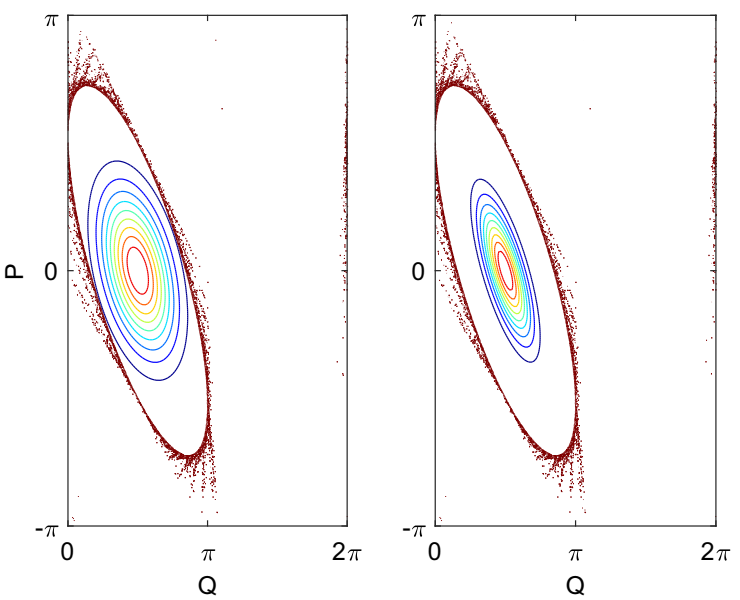

Fig. 3 Husimi (left) and Wigner (right) functions for the same initial state with the following parameters: $\sqrt{D}=1.3, \varphi=$ $1.005, \alpha=-2.1, \theta_{1}=\pi, \theta_{2}=\frac{3}{2} \pi$. The dots represent the sticking area of classical AM, while ellipses are contour lines of corresponding functions, $P=P_{m} \bmod 2 \pi$

and

$\Sigma^{2}=\frac{\hbar}{2} \frac{\alpha \cos \varphi-i \sin \varphi}{\cos \varphi-i \alpha \sin \varphi}$.

We can see that the transformation from $\psi(Q)$ to $\psi_{\varphi}(Q)$ is equivalent to replacing $D$ with a complex parameter. We can say that the function $\psi_{\varphi}(Q)$ is a product of Gaussian and linear chirp. The formula above can also be used in the cylinder case if the Gaussian function is sufficiently narrow, i.e., if its width is much smaller than $2 \pi$.

In Fig. 4, we present the Husimi function for the initial state (rotated Gaussian) localized in AM in the cell described by the Cartesian product: $[0,2 \pi) \times[-\pi, \pi)$ and five of its iterations for $n=1,2,10,100,1000$. We can observe that the Husimi functions of these iterations are localized in AMs in cells of phase space described by $[0,2 \pi] \times[(2 n-1) \pi,(2 n+1) \pi]$, where $n$ is the iteration number. Moreover, the form of the Husimi function changes slightly. However, there exists tunneling from AM to the remaining phase space. In order to investigate the properties of this tunneling, we calculate the probability $W(n)=\sum_{i}\left|\psi\left(P_{i}\right)\right|^{2}$, where $P_{i} \in[(2 n-1) \pi,(2 n+1) \pi]$ at each iteration of the initial state. When proceeding in this way, however, we are calculating rather the escape rate from the cell with AM than from AM itself.

The results of numerical calculations for the chosen value of $D$ and $\hbar$ are presented in Figs. 5 and 6. In the first one, we present Wigner functions of the initial state 
Fig. 4 Husimi function of the states for various iteration steps: $n=0,1,2,10,100,1000$
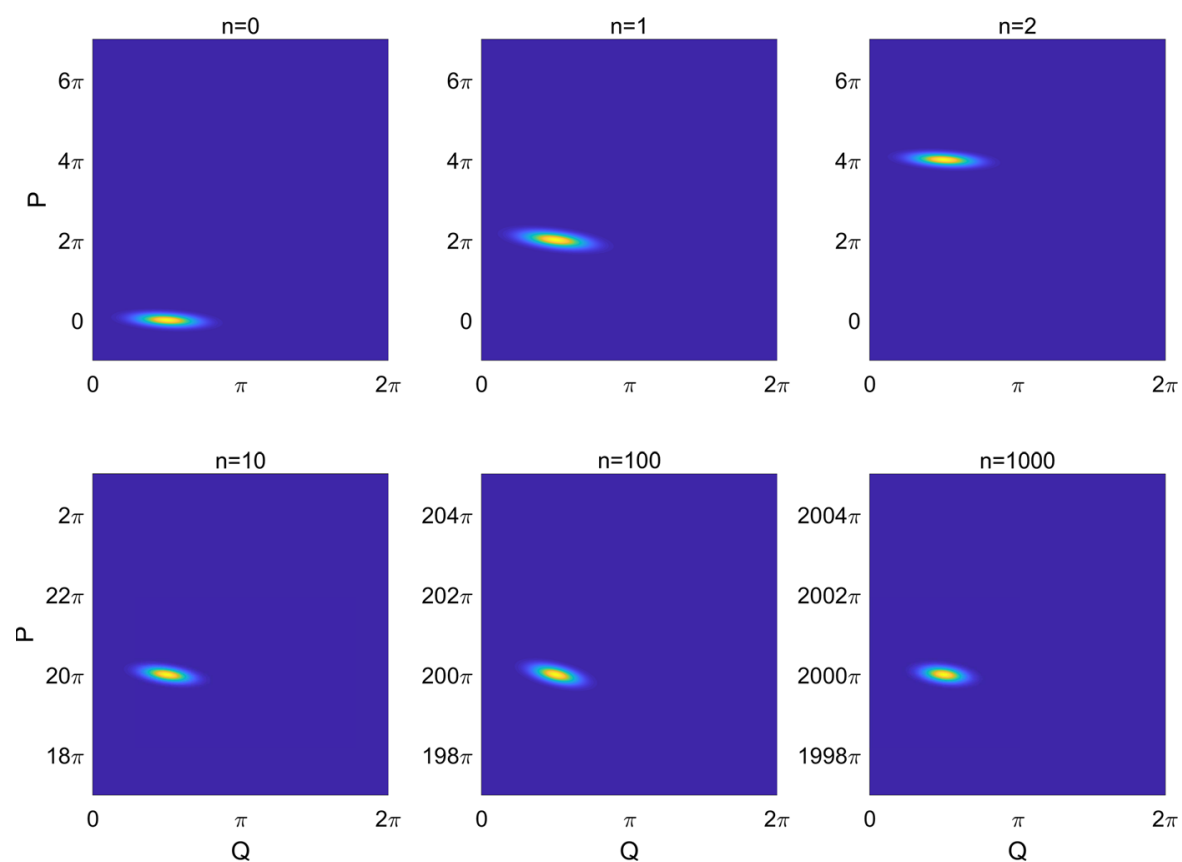

$W(n) \sim \exp (-\beta n)$

with $\beta=9.46 \times 10^{-7}$. Such a good fit was obtained for the same initial state as in the previous figure. On the right side of Fig. 6, where the initial state has larger values of parameter $D$, we obtain a good fit to the straight line for results with $n>5000$. A value of $\beta=9.44 \times 10^{-7}$ was estimated for iterations with $n>30000$. Such a calculated value of the $\beta$ parameter is practically the same as in the previous figure $(D=0.358)$.

Similar simulations can be performed when the Husimi function is well localized outside of the AM, in the chaotic part of phase space. In contrast to the situation presented in Fig. 4, the initial state gradually fills the chaotic part of phase space leaving intact regions where AMs are localized, which is clearly visible for the iteration $n=3$ in Fig. 7.

We have also studied the asymptotics of numerically obtained densities, where a maximum of Husimi functions lay outside the accelerator mode. In Fig. 8, we present an evolution of a wave function amplitude $|\Psi(P, t)|$ in momentum representation. The amplitude has a maximum, which moves in the direction of the negative $P$-values with constant velocity. At the same time, it possesses a long tail for positive $P$-values with the front which moves with constant velocity as well (the front jumps by $2 \pi$ at every iteration). In order to 

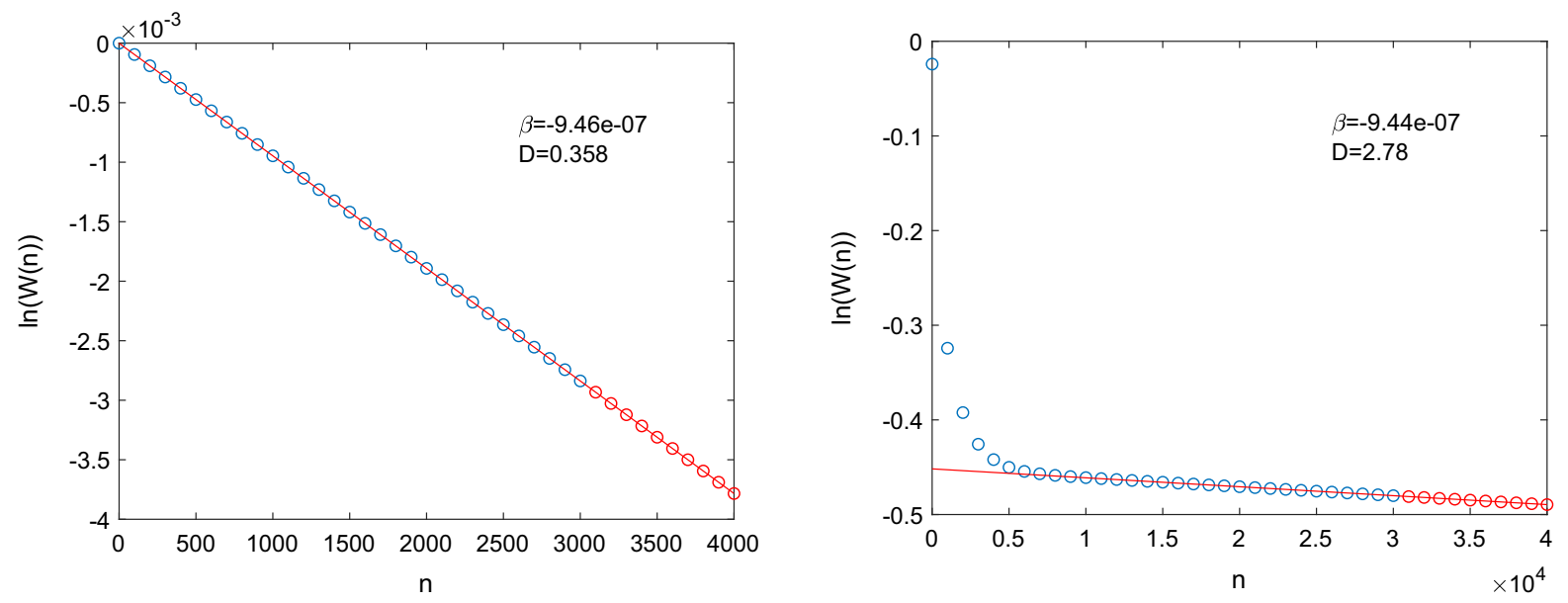

Fig. 6 Logarithm of probability as a function of iteration step (marked by circles) and straight line fitted to the last ten points

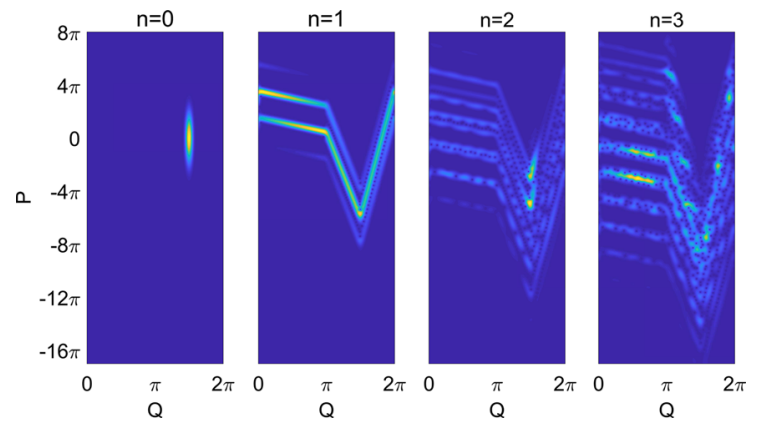

Fig. 7 Husimi function in selected iterative steps. The initial state $n=0$ is a Gaussian one with parameters $D=0.1 Q_{0}=\frac{3 \pi}{2}$ and $P_{0}=0$. For $n=1,2,3$, we have the next quantum iterative steps. We use following parameters: $I=1.0000018, K=1$, $a_{1}=-2,1, \theta_{1}=\pi, \theta_{2}=\frac{3}{2} \pi, \hbar=\frac{\pi}{64}, T=1$ and the momentum range $[-8 \pi, 8 \pi]$

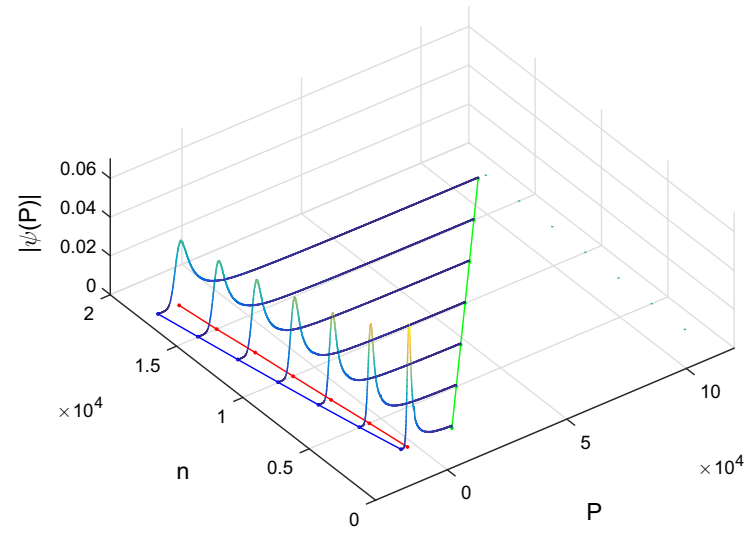

Fig. 8 Amplitude $|\Psi(n, P)|$ of a wave function in momentum representation for selected iteration steps $n$ verify the nature of this tail, we consider a state after a large number of iterations as is presented in Fig. 9 on the left side. The value of the wave function amplitude is drawn in two scales, which can be seen on the right side of this figure. The upper figure presents a linear fit to the results in log-log scale that has a high coefficient of determination. It is a strong numerical argument that the asymptotics of the fragment of $|\Psi(P)|$ under consideration has an inverse power character as in the classical case (see Fig. 2 in [5]).

Taking into account the results of our numerical calculations, we can state that basic properties of quantum distribution retain the properties of the classical model (despite quite a large Planck constant). The AMs still have an influence on quantum evolution as in the classical model. Both in the classical picture and the quantum one, densities have a pronounced maximum for some values of momentum while for larger values of momentum they have inverse power asymptotics.

\section{Further modifications of the standard map}

Replacing the function $f(Q)$ with a four-part piecewise linear continuous periodic function for which $\left\langle P_{n+1}-\right.$ $\left.P_{n}\right\rangle=0$. For certain parameter values, we can observe the emergence of two kinds of AM in phase space. In one type of $\mathrm{AM}$, the $\mathrm{P}$ value grows approximately linearly during successive iterations. In the other type of AM, the movement goes in the opposite direction. Both types of AM are presented in Fig. 10. 
Fig. 9 The picture on the left presents amplitude $|\Psi(P)|$ in momentum representation for 16,000 iterations. In the pictures on the right, the upper graph is a fragment of distribution in both logarithmic scales with linear fitting and a slope value of -1.78 ; the bottom graph presents results in a linear-logarithmic scale for the same range of $P$ values as in the upper graph

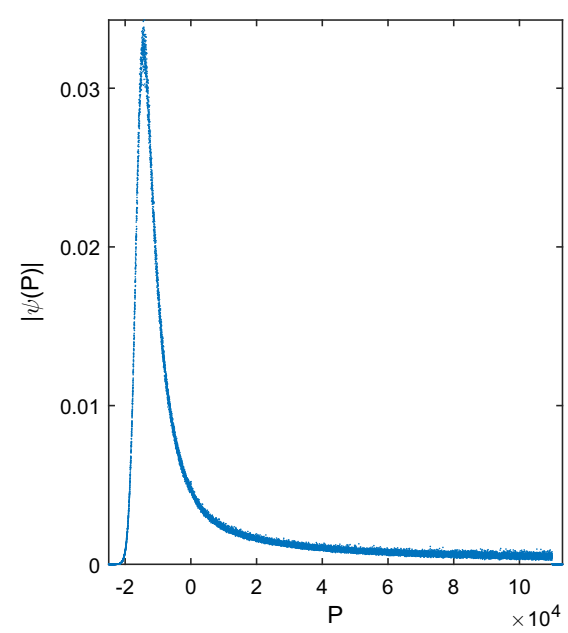

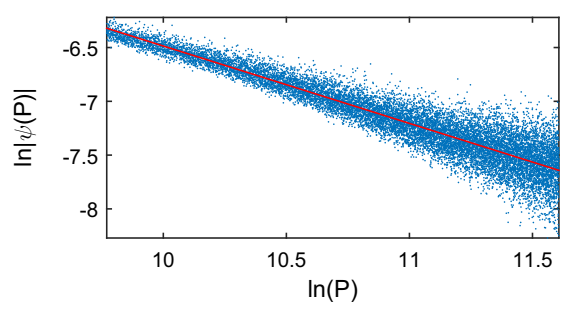

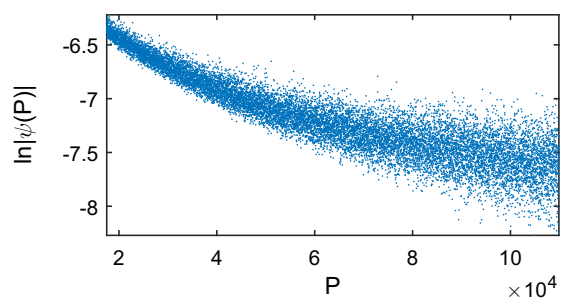

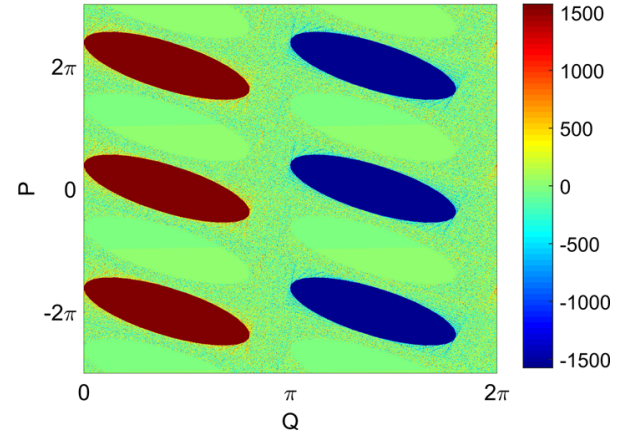

Fig. 10 Phase space of a modified standard map, where $f(Q)$ have four piecewise linear parts. Color represents the velocity changes of momentum

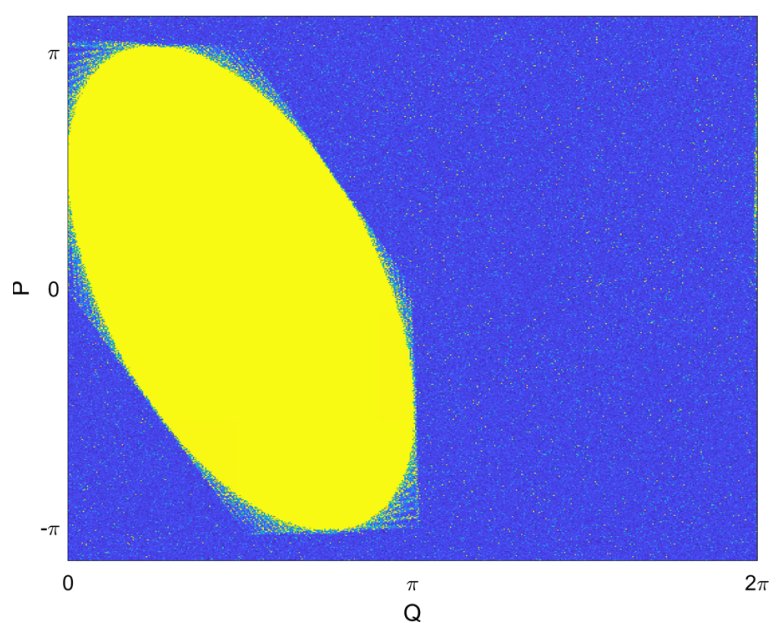

We can also modify function (5) requiring that it be differentiable, i.e., that it be a function of class $C^{n}$. It can be realized by joining its adjacent linear parts by a polynomial of order $n+1$ on some small intervals of length $D$. All coefficients of the linear and polynomial parts can be uniquely determined by periodicity, continuity of functions, and their derivatives. The proper choice of model parameters allows us to obtain AM in the phase space (see Fig. 11). In the limit $D \rightarrow 0$, this function becomes $f(Q)$ defined by formula (5).

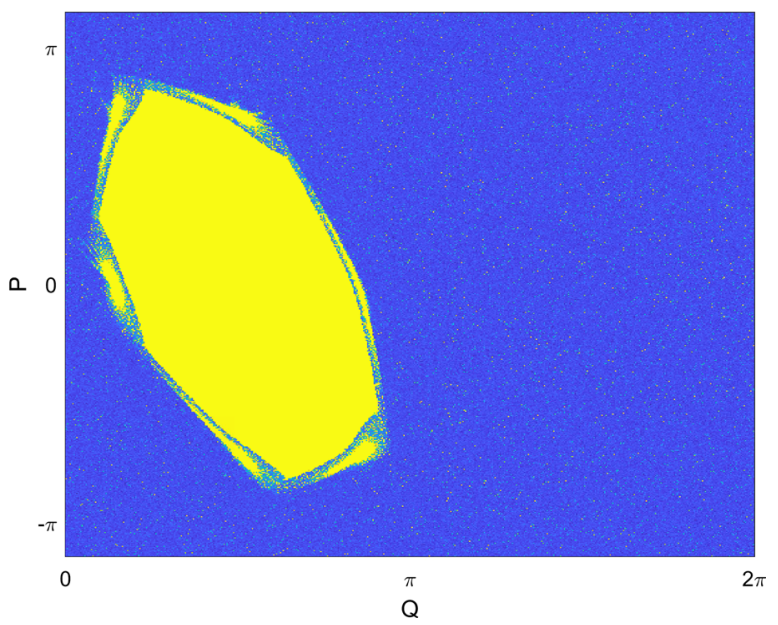

Fig. 11 AM for another modification of SM 


\section{Conclusions}

We have presented a model that is a modification of the $\mathrm{SM}$. The kicking function is a smooth piecewise linear function parametrized by one parameter (besides position of three intervals). For properly chosen parameters, there exist large AMs. This feature simplifies the numerical analysis of the quantum mapping. Our analysis shows that AM and its stickiness property are retained in the quantum picture. The quantum evolution of states localized in AM follows classical evolution, i.e., the quantum map translates the localized state in $\mathrm{AM}$ to the next $\mathrm{AM}$, practically without changing its Husimi function, with the exception of very small tunneling to the rest of phase space. Quantum probability density in momentum representation preserves many essential features of classical momentum density. Numerical results show that states which are localized in the chaotic part of phase space delocalize under the influence of quantum mapping in a manner similar to the classical case. For a sufficiently long time (a number of a map iterations), density has an inverse power asymptotic for large momentum with a characteristic front moving with constant velocity. This model is relatively easy to analyze when instead of kick, i.e., $\delta$-time dependence, we use a continuous periodic function. In such a case, one has to apply Floquet theory.

\section{Compliance with ethical standards}

Conflict of interest The authors declare that there is no conflict of interests regarding the publication of this paper.

Open Access This article is distributed under the terms of the Creative Commons Attribution 4.0 International License (http:// creativecommons.org/licenses/by/4.0/), which permits unrestricted use, distribution, and reproduction in any medium, provided you give appropriate credit to the original author(s) and the source, provide a link to the Creative Commons license, and indicate if changes were made.

\section{References}

1. Ott, E.: Chaos in Dynamical Systems. Cambridge University Press, Cambridge (1993)

2. Gaspard, P.: Chaos. Scattering and Statistical Mechanics. Cambridge University Press, Cambridge (1998)

3. Zaslavsky, G.M.: Hamiltonian Chaos and Fractional Dynamics. Oxford University Press, New York (2005)

4. Chirikov, B.V.: A universal instability of many-dimensional oscillator systems. Phys. Rep. 52(5), 263-379 (1979)

5. Weber, P., Pepłowski, P.: Gaussian diffusion interrupted by Lévy walk. J. Stat. Phys. Theory Exp. 2016(10), 103202 (2016)

6. Karney, C.F.F.: Long-time correlations in the stochastic regime. Physica D 8(3), 360-380 (1983)

7. Chirikov, B.V., Shepeliansky, D.L.: Correlation properties of dynamical chaos in Hamiltonian systems. Physica D 13(3), 395-400 (1984)

8. Ishizaki, R., Horita, T., Kobayashi, T., Mori, H.: Anomalous diffusion due to accelerator modes in the standard map. Prog. Theor. Phys. 85, 1013-1022 (1991)

9. Schlunk, S., d'Arcy, M.B., Gardiner, S.A., Cassettari, D., Godun, R.M., Summy, G.S.: Signatures of quantum stability in a classically chaotic system. Phys. Rev. Lett. 90(5), 054101 (2003)

10. Buchleitner, A., d'Arcy, M.B., Fishman, S., Gardiner, S.A., Guarneri, I., Ma, Z.Y., Rebuzzini, L., Summy, G.S.: Quantum accelerator modes from the Farey tree. Phys. Rev. Lett. 96(6), 164101 (2006)

11. Ma, Z.Y., d'Arcy, M.B., Gardiner, S.A.: Gravity-sensitive quantum dynamics in cold atoms. Phys. Rev. Lett. 93(16), 164101 (2004)

12. Wimberger, S., Guarneri, I., Fishman, S.: Classical scaling theory of quantum resonances. Phys. Rev. Lett. 92(8), 084102 (2004)

13. Berry, M.V.: Semi-classical mechanics in phase space: a study of Wigner's function. Philos. Trans. R. Soc. Lond. 287(1343), 237-271 (1977)

14. Chism, W., Timberlake, T., Reichl, L.E.: High harmonic generation in systems with bounded chaos. Phys. Rev. E 58(2), 1713-1723 (1998)

15. Frahm, K.M., Fleckinger, R., Shepelyansky, D.L.: Quantum chaos and random matrix theory for fidelity decay in quantum computations with static imperfections. Eur. Phys. J. D 29(1), 139-155 (2004) 